\title{
Balancing Image Quality and Energy Consumption in Visual Sensor Networks
}

\author{
Kit-Yee Chow, King-Shan Lui and Edmund Y. Lam \\ Department of Electrical and Electronic Engineering \\ The University of Hong Kong \\ Pokfulam Road, Hong Kong, China
}

\begin{abstract}
Applications of wireless sensor networks have attracted a lot of attention recently. Cameras are installed in various locations of a wide area to capture images of targeted objects. Because of constraints in computational capability in these distributed cameras, it may not be feasible to analyze these images in the sensors but they have to be transmitted to a centralized server hop by hop through the sensor network. To reduce the energy used in transmission, the size of the images should be kept small by applying a large compression ratio, which may degrade image quality. This paper studies the tradeoff between image quality and energy consumption. We study the scenario that a number of camera-equipped sensors are taking pictures of the same object, and the pictures of adjacent cameras may overlap. We demonstrate that by allowing intermediate sensors to process the images and combine the overlapping portions, the total energy spent on transmission is reduced subject to a certain degradation in image quality. The tradeoff between image quality and energy consumption of different routing presents an important study on the practicability of visual sensor networks.
\end{abstract}

\section{INTRODUCTION}

A wireless sensor network consists of thousands of sensors that span a large geographical region. Research and development in wireless sensor networks are becoming increasingly widespread due to their low cost and low maintenance in deployment. These sensors are able to communicate with each other to collaboratively detect objects, collect information, and transmit messages. Sensor networks have become an important technology especially for environmental monitoring, military applications, disaster management, etc [1][2]. A sensor is a very small device and the battery inside is not likely to be rechargeable. This limitation in energy puts extra constraints in the operations of a sensor. In order to prolong its lifetime, a sensor should carefully utilize its energy. Message between transmission has been shown to be the major source of energy dissipation. To save energy used in transmission, the size of the messages to be transmitted has to be reduced. If the content of a message is an image, a reduction in message size often implies a reduction in image quality as well. Hence, there are tradeoffs between reducing energy consumption and maintaining a good image quality, and this paper studies a way to balance the two conflicting goals.

A sensor node can reduce the energy spent in transmission by combining the data it receives from neighbors together before transmitting it out. A sensor node can "combine" a few messages into one by calculating the average of the messages.
Energy saved in this way is called data aggregation. The problem of finding optimal data aggregation has been proved to be NP-hard [3]. Some mechanisms have been developed to aggregate simple scalars [4][5][6], but only a few of them study the employment of aggregation in image transmission. Ref [7] shows that applying maximum compression before transmission may not always minimize energy used. The authors then develop a heuristic for selecting a good compression level. Ref [8] studies distributed image compression that the whole compression process of a single image is distributed among different groups of sensor nodes. This approach does not decrease the total energy needed, but the maximum energy needed in a sensor is reduced. [9] also studies distributed image compression. Overlapping areas of images are identified and sensors send a low-resolution version of these areas for the receiver to reconstruct the overlapped blocks in highresolution. None of the work mentioned above considers the effect of using different paths in transmitting the images.

In this paper, we study the tradeoff between transmission energy consumption and image quality when different routing paths are used. We demonstrate that by allowing an intermediate sensor to combine the overlapping portions of the images it receives, transmission energy is saved by sacrificing some image quality. Our study also shows that different paths can result in different image quality and energy consumption. The results of the studies are very helpful in developing distributed algorithms in visual sensor networks for efficient image transmission. The rest of the paper is organized as follows: Section II presents the network model. Section III presents the simulation results. Finally, we conclude our paper in Section IV.

\section{Problem Statement}

\section{A. Network Model}

To facilitate our discussion, we consider a simple scenario where three cameras, $C_{1}, C_{2}$ and $C_{3}$, are taking pictures that contain same object. Images have to be sent to a server $\mathrm{S}$ for analysis. Sensor $P_{1}$ is adjacent to $C_{1}$ and $C_{2}$ while sensor $P_{2}$ is adjacent to $C_{2}$ and $C_{3}$. The cameras have to send their images to either $P_{1}$ or $P_{2}$, which then relay the images to $\mathrm{S}$. There may be more than one hop between $P_{1}$ or $P_{2}$ to $\mathrm{S}$. Apart from processing functions, these sensors are able to perform image processing functions. For example, $P_{1}$ and $P_{2}$ can decompress images sent by the cameras and recompress 


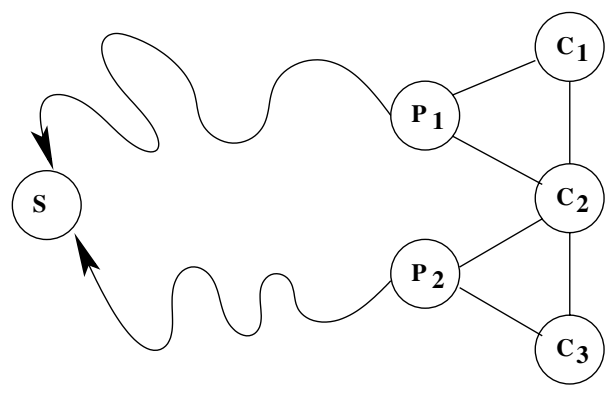

Fig. 1. Network Model

them after processing. The images sent by $P_{i}$ will go hop by hop to $S$. The paths are represented as wavy arrows in Figure 1. We assume that the intermediate nodes on the path from $P_{i}$ to $S$ are equipped with communication devices only. They are responsible for sending out the images and do not have image processing functions.

\section{B. Image Compression}

We assume that JPEG is used for compressing images. Both camera nodes and intermediate nodes have the capability of compressing images and with different quality level. Quality level is the parameter to control the compression amount. In JPEG compression, to reduce the quality we can reduce the number of quantization levels. This results in down-sampling. By doing this, we require less number of bits to store each pixel intensity. This way compression is achieved. The higher the quality level, the better the image quality but with a larger file size. The quality level of cameras is set to be $x$ while the quality level of intermediate nodes is set to be $y$, where $x<y$. As cameras have to spend energy in capturing pictures, it is desirable to reduce the energy spent in transmission. Thus, we set the quality level of cameras to be smaller so that images produced will be smaller in order to reduce the transmission load. $x$ is smaller than $y$ because the transmission load in intermediate nodes is less than that in camera nodes. We can tolerate a lower degree of compression in order to have a better image quality.

\section{Image Transmission}

We denote the compressed image captured by $C_{i}$ as $I_{i}$. Data size of image $I_{i}$ after compression is denoted as $\left|I_{i}\right|$. Since the network spans over a large area, the captured images usually overlap with the adjacent images. For example, suppose $I_{1}$ and $I_{2}$ overlap with each other in the region $I_{1} \cap I_{2}$. When $P_{1}$ receives these two images, it can combine them to form $I_{1+2}$. $I_{1}$ and $I_{2}$ will be decompressed, processed, and recompressed to form a new version of that region. The duplicate information $I_{1} \cap I_{2}$ will be averaged to reduce noise. We assume that the computational cost to perform averaging is negligible compared to the transmission energy and compression energy.

There are six different ways of transmission as shown in Figure 2 [10]. We assume that a single node can combine at most two images. For example, in Method A, $I_{1}$ and $I_{2}$ are sent to $P_{1}$ while $I_{3}$ is sent to $P_{2}$. Upon receiving $I_{1}$ and $I_{2}$, which are compressed using quality level $x, P_{1}$ decompresses the overlapping portion in each image and then recombines them. The non-overlapping regions in both images remain unchanged. The combined version is then compressed with a quality level of $y$. As $P_{2}$ receives only one image, $I_{3}$, it simply sends it out to $S$ without processing it.

It is also possible that data fusion can be done in camera nodes as in Methods $\mathrm{C}$ to $\mathrm{F}$. In Method $\mathrm{C}, C_{2}$ sends its image to $C_{1}$. To form $I_{1} \cap I_{2}$ upon receiving $I_{2}, C_{1}$ only needs to decompress the overlapping portion of $I_{2} . I_{1}$ is not decompressed since $C_{1}$ has the raw data of $I_{1}$. The nonoverlapping region of $I_{2}$ remains unchanged. After that, $C_{1}$ will compress the new version of $I_{1} \cap I_{2}$ with quality level of 15. As in Method A, $P_{2}$ receives only one image, $I_{3}$, it simply relays the image without any processing. One may notice that the combined overlapping portions will have quality level of $y$ in Methods A and B. While the quality level of the combined versions in Methods $\mathrm{C}$ to $\mathrm{F}$ will be $x$.

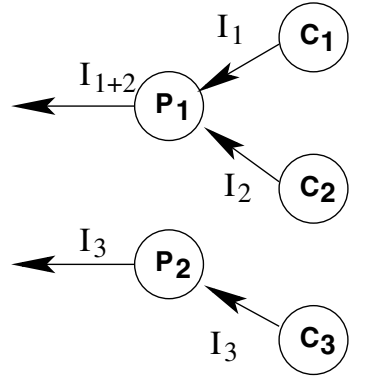

Method A

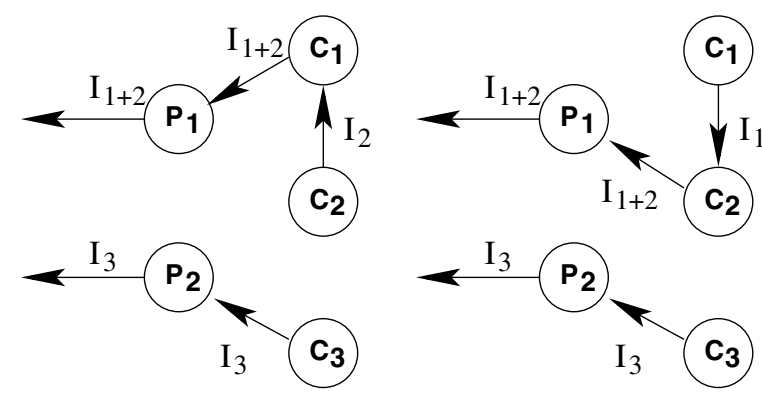

Method C

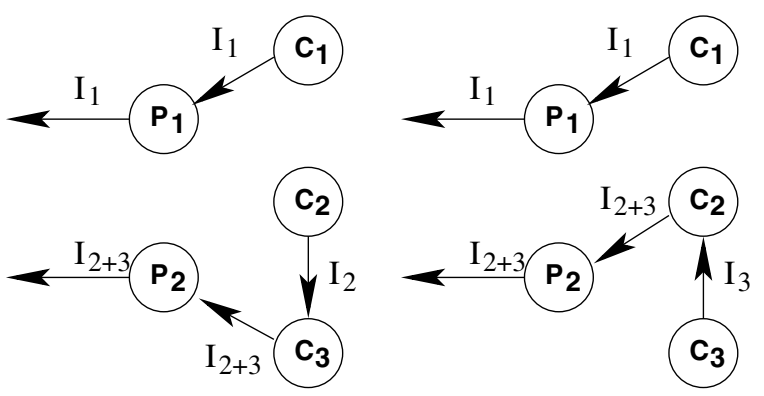

Method E

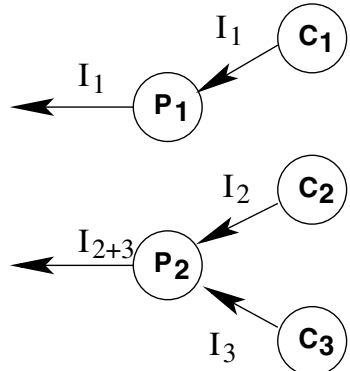

Method B
Fig. 2. Six different ways of transmission 


\section{Energy consumption}

Both image transmission and image processing require energy. The energy consumption for transmission depends on the image size and the number of hops that it traverses. We assume that the energy needed to traverse each hop is the same for the same image. The energy needed in processing is the sum of the energy spent in image compression and decompression in each node.

Let $c\left(I_{i}\right)$ be the energy needed to compress image $I_{i}, t\left(I_{i}\right)$ be the energy needed to transmit $I_{i}$ to a neighbor node, $d\left(I_{i}\right)$ be the energy needed to decompress image $I_{i}$ and $h\left(P_{i}\right)$ be the number of hops on the path from $P_{i}$ to $S$. For example, in Method A, the total transmission energy is

$$
T=t\left(I_{1}\right)+t\left(I_{2}\right)+t\left(I_{3}\right)+h\left(P_{1}\right) * t\left(I_{1+2}\right)+h\left(P_{2}\right) * t\left(I_{3}\right) .
$$

And the energy needed in processing is the sum of energy spent in compression and decompression in each node, which is

$$
P=c\left(I_{1}\right)+c\left(I_{2}\right)+c\left(I_{3}\right)+2 * d\left(I_{1} \cap I_{2}\right)+c\left(I_{1} \cap I_{2}\right) .
$$

In this paper, we assume compression and decompression consume the same amount of energy under JPEG regardless of the quality level. So the processing energy in Method A becomes

$$
P=c\left(I_{1}\right)+c\left(I_{2}\right)+c\left(I_{3}\right)+3 * c\left(I_{1} \cap I_{2}\right) .
$$

In Method $\mathrm{C}$, the total transmission energy will be:

$$
T=t\left(I_{2}\right)+t\left(I_{3}\right)+\left(h\left(P_{1}\right)+1\right) * t\left(I_{1+2}\right)+h\left(P_{2}\right) * t\left(I_{3}\right) .
$$

As mentioned above, $C_{1}$ only needs to decompress the overlapping portion of $I_{2}$. So the processing energy in Method $\mathrm{C}$ becomes

$$
\begin{aligned}
P & =c\left(I_{1}-I_{1} \cap I_{2}\right)+c\left(I_{2}\right)+c\left(I_{3}\right)+2 * c\left(I_{1} \cap I_{2}\right) \\
& =c\left(I_{1}\right)+c\left(I_{2}\right)+c\left(I_{3}\right)+c\left(I_{1} \cap I_{2}\right) .
\end{aligned}
$$

The energy consumption in Methods B to F can be calculated in a similar manner. It can be observed that determining which method would consume the least amount of energy is not trivial. If $h\left(P_{1}\right)>h\left(P_{2}\right)$, the transmission energy for methods $\mathrm{B}, \mathrm{E}$ and $\mathrm{F}$ will be smaller than the others. Since $t\left(I_{a+b}\right)$ is always larger than $t\left(I_{a}\right)$ or $t\left(I_{b}\right)$, the energy consumption greatly depends on the geographical distribution of sensors.

\section{Simulation}

In this section, we present our simulation results that show the tradeoff between energy consumption and image quality. The simulation results are generated by using MATLAB. The size of the raw image captured by each camera is $500 \times 500$. The compression quality levels $x$ and $y$ are set to be 15 and 30 respectively. And four different pictures are used for calculating the average simulation results.

Depending on applications, the energy consumed in transmitting versus compressing or decompressing the same amount of image data can vary substantially [7]. Therefore, in each scenario, we examine the transmission energy required for different methods under various ratios of transmission energy to compression energy.

We use total message size to measure the total transmission energy. The compression energy is measured by the file size of the image to be compressed/decompressed. On the other hand, the image quality is measured by the mean square error (MSE) of the image obtained in $S$. The smaller the mean square error, the better the image quality. MSE is defined as

$$
M S E=\frac{1}{Q} \sum_{k=1}^{Q}(I(k)-\hat{I}(k))^{2},
$$

where $I(k)$ is the original image, $\hat{I}(k)$ is the reconstructed image at $S$, and $\mathrm{Q}$ is the total number of pixels.

Apart from Methods $\mathrm{A}$ to $\mathrm{F}$ as mentioned above, we also simulate the scenario that no processing is done in the intermediate sensor nodes. That is, $P_{1}$ and $P_{2}$ simply relay the images. The final server $\mathrm{S}$ can enhance the final image quality by averaging the overlapping regions $I_{1} \cap I_{2}$ and $I_{2} \cap I_{3}$. As mentioned before, the camera nodes and processing nodes are using different compression quality level. (i.e. quality level of $C_{i}=15$, quality level of $p_{i}=30$ )

We assume $P_{1}$ and $P_{2}$ have equal path length. That is, the number of hops on the path from $P_{1}$ to $S$ is equal to that of $P_{2}$. Energy used with different number of hops are measured. Although $\left|I_{1}\right|,\left|I_{2}\right|$ and $\left|I_{3}\right|$ are of the same size, $\left|I_{1} \cap I_{2}\right|$ and $\left|I_{2} \cap I_{3}\right|$ may not be the same. In this section, we consider four different overlapping ratios:

1) $\left|I_{1} \cap I_{2}\right|=\left|I_{2} \cap I_{3}\right|=0.5|I|$

2) $\left|I_{1} \cap I_{2}\right|=\left|I_{2} \cap I_{3}\right|=0.3|I|$

3) $\left|I_{1} \cap I_{2}\right|=0.3|I|,\left|I_{2} \cap I_{3}\right|=0.7|I|$

4) $\left|I_{1} \cap I_{2}\right|=\left|I_{2} \cap I_{3}\right|=0.25|I|$

The results of all cases are similar. Cases 1 to 4 have similar trends in both energy consumption plot and MSE plot, only the most significant plots are shown.

Figures 3 and 4 are the results of Case $3\left(\left|I_{1} \cap I_{2}\right|=0.3|I|\right.$ and $\left.\left|I_{2} \cap I_{3}\right|=0.7|I|\right)$. Let $E_{c}$ be the compression energy per 
byte and $E_{t}$ be the transmission energy per byte. The x-axis is representing $\frac{E_{t}}{E_{c}}$. Let $\mathrm{T}$ be the total transmission load and $\mathrm{P}$ be the total compression load. The total energy consumption equals

$$
E_{\text {total }}=T \times E_{t}+P \times E_{c}
$$

The y-axis is representing the normalized energy consumption, that is $\frac{E_{\text {total }}}{E_{c}}$.

When the number of hops equals to one, images will be sent to $S$ directly through $P_{1}$ and $P_{2}$. Figure 3 shows that the energy consumption in the dummy case (i.e. without processing) is always the least. When the number of hops equals 15 , $P_{1}$ and $P_{2}$ are far away from the server $S$, the method which consumes the least amount of energy is no longer always the dummy case. As shown in Figure 4, Methods C, D, E and F consume less energy when the ratio of transmission energy to compression energy is about $10^{0.4}$.

$I_{1}, I_{2}, I_{3}$ are each $500 \times 500$ in size. For case $1\left(\left|I_{1} \cap I_{2}\right|=\right.$ $\left|I_{2} \cap I_{3}\right|=0.5|I|$ ), the final reconstructed image will be $500 \times 1000$ in size. The image is divided into 4 rectangular blocks, where each block is $500 \times 250$ in size. The mean square error of the second block of different methods are compared with the mean square error of the dummy case. The result is shown in Figure 5. It can be observed that image qualities of methods A, C and D are worse. This is because the data in this block has undergone decompression and recompression in the intermediate node $P_{1}$ in methods $\mathrm{A}, \mathrm{C}$ and $\mathrm{D}$. The result will be totally different if the third block of data is compared instead of the second block. Methods B, E and F will be worse, since the data in this block is processed in $P_{2}$.

Figure 6 shows the overall MSE of the whole reconstructed image in Case 1. The overall MSE is the average value of the MSEs of each block of data. It can be observed that the image qualities of methods A to F vary. None of them is as good as the dummy case. Errors have been introduced in the process of decompression and recompression during the data aggregation at intermediate nodes.

\section{CONCLUSION}

In this paper, we show that there is a tradeoff between energy consumption and image quality in multi-hop visual sensor networks. When the cameras are far away from the server, it is better to process the overlapping regions of images by intermediate sensors to reduce energy overhead. On the other hand, if the cameras are very near the server, processing of intermediate nodes may not bring significant benefits. Under different ratios of transmission and compression energy, overlapping ratios, and network path length, we should perform different routing in order to balance the tradeoff between image quality and energy consumption.

In this paper, we considered a simple network consisting three cameras only. In reality, there may be thousands of camera nodes in the network. We may further balance the tradeoff with the concept of region of interest [11]. We may want to perform data aggregation on the data outside the region of interest, so that overhead is reduced and the image quality of the region of interest can be maintained.

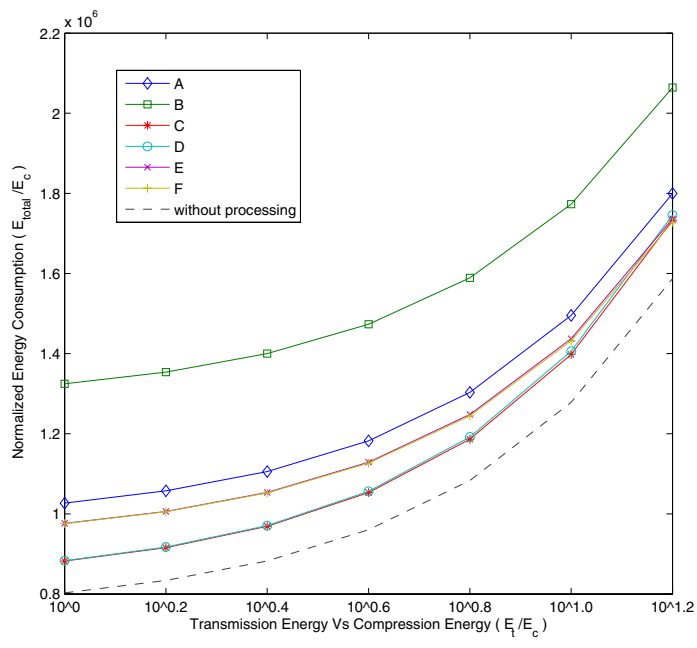

Fig. 3. Case 3 , no. of hops $=1$.

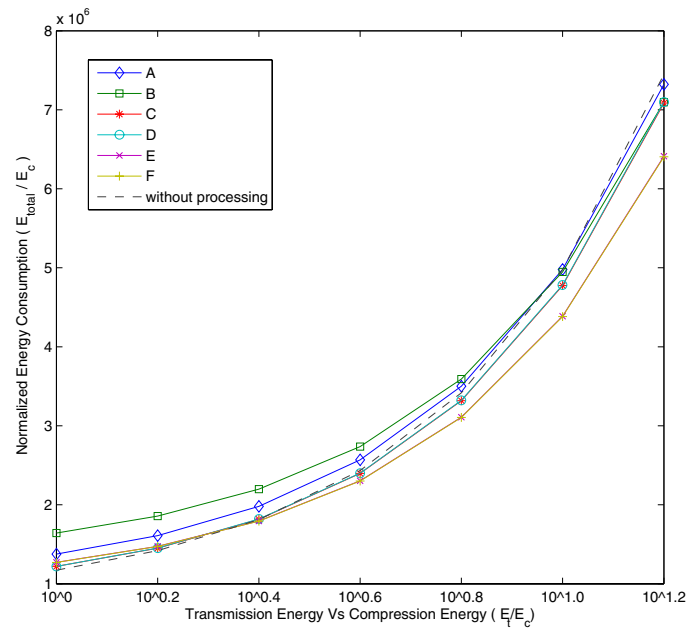

Fig. 4. Case 3, no. of hops $=15$.

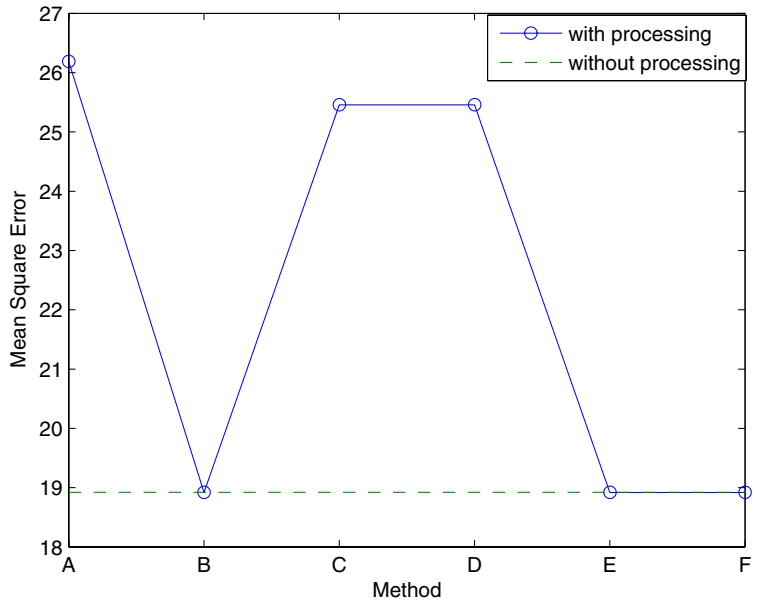

Fig. 5. Block 2 Mean Square Error (Case 1) 


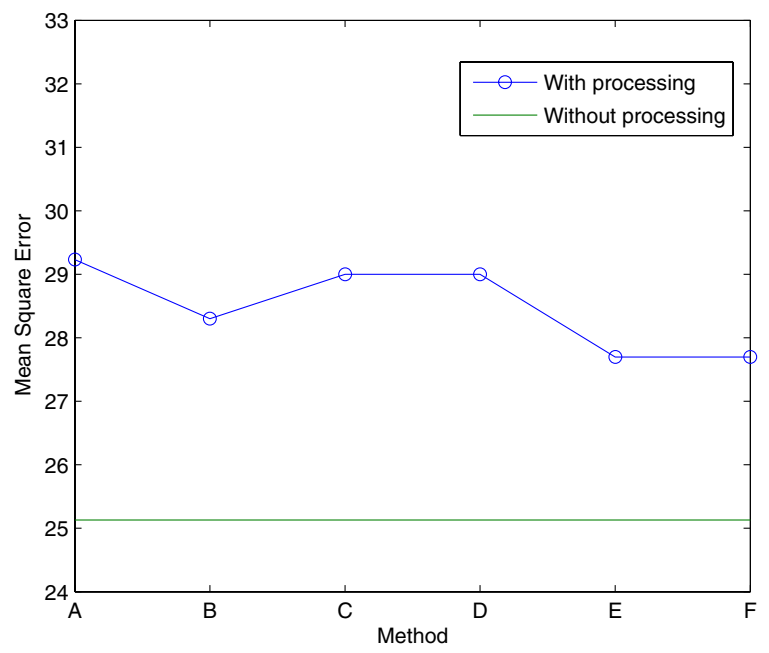

Fig. 6. Case 1, overall mean square error.

\section{REFERENCES}

[1] D. Estrin, D. Culler, K.Pister, and G.Sukatme, "Connecting the physical world with pervasive networks," IEEE Pervasive Computing, pp. 59-69, 2002.

[2] G.J.Pottie and W.J.Kaiser, "Wireless integrated network sensors," Communications of the ACM, vol. 43, no. 5, pp. 51-58, 2000.

[3] B. Krishnamachari, D. Estrin, and S.Wicker, "The impact of data aggregation in wireless sensor networks," International Workshop of Distributed Event Based Systems, pp. 575-578, 2002.

[4] M.Ding, X.Cheng, and G.Xue, "Aggregation tree constuction in sensor networks," IEEE Vehicular Technology Conference, pp. 2168-2172, 2003.

[5] N.Shrivastava, C.Buragohain, and D.Agrawal, "Medians and beyond: New aggregation techniques for sensor networks," ACM SenSys, 2004.

[6] S.Nath, P.B.Gibbons, S.Seshan, and Z.R.Anderson, "Synopsis diffusion for robust aggregation in sensor networks," ACM SenSys, 2004.

[7] H.Wu and A.A Abouzeid, "Power aware image transmission in energy constrained wireless netowrks," IEEE Symposium on Computers and Communications (ISCC), pp. 202-207, 2004.

[8] H.Wu and A.A Abouzeid, "Energy efficient distributed JPEG2000 image compression in multihop wireless networks," Workshop on Applications and Services in Wireless Networks (ASWN), 2004.

[9] R.Wagner, R.Nowak, and R.Baraniuk, "Distributed image compression for sensor networks using correspondence analysis and super-resolution," IEEE International Conference on Image Processing, pp. 597-600, 2003.

[10] King-Shan Lui and Edmund Y. Lam, "Image transmission in sensor networks," Workshop on Signal Processing Systems (SiPS), 2005.

[11] David S. Taubman and Michael W. Marcellin, JPEG2000: image compression fundamentals, standards, and practice, Kluwer Academic Publishers, 2002. 